\title{
Podocyte Loss and Progressive Glomerular Injury in Type II Diabetes
}

\author{
Maria Enrica Pagtalunan, ${ }^{\star \ddagger}$ Peter L. Miller, ${ }^{\star \ddagger}$ Sara Jumping-Eagle, ${ }^{\star \ddagger}$ Robert G. Nelson, ${ }^{\S}$ Bryan D. Myers, ${ }^{* \ddagger}$ \\ Helmut G. Rennke, Norman S. Coplon, ${ }^{\star \ddagger}$ Limei Sun, ${ }^{\star \ddagger}$ and Timothy W. Meyer ${ }^{\star \ddagger}$ \\ *Department of Medicine, VA Palo Alto Healthcare System, Stanford, California 94305; ${ }^{*}$ Stanford University, Stanford, California 94305 ; \\ ${ }^{\S}$ Phoenix Epidemiology and Clinical Research Branch, National Institute of Diabetes and Digestive and Kidney Diseases, Phoenix, \\ Arizona 85014; and "Department of Pathology, Brigham and Women's Hospital and Harvard Medical School, Boston, Massachusetts \\ 02115
}

\begin{abstract}
Kidney biopsies from Pima Indians with type II diabetes were analyzed. Subjects were classified clinically as having early diabetes $(n=10)$, microalbuminuria $(n=17)$, normoalbuminuria, despite a duration of diabetes equal to that of the subjects with microalbuminuria $(n=12)$, or clinical nephropathy $(n=12)$. Subjects with microalbuminuria exhibited moderate increases in glomerular and mesangial volume when compared with those with early diabetes, but could not be distinguished from subjects who remained normoalbuminuric after an equal duration of diabetes. Subjects with clinical nephropathy exhibited global glomerular sclerosis and more prominent structural abnormalities in nonsclerosed glomeruli. Marked mesangial expansion was accompanied by a further increase in total glomerular volume. Glomerular capillary surface area remained stable, but the glomerular basement membrane thickness was increased and podocyte foot processes were broadened. Broadening of podocyte foot processes was associated with a reduction in the number of podocytes per glomerulus and an increase in the surface area covered by remaining podocytes. These findings suggest that podocyte loss contributes to the progression of diabetic nephropathy. (J. Clin. Invest. 1997. 99: 342-348.) Key words: glomerulus - proteinuria • mesangium • sclerosis • epithelial cell
\end{abstract}

\section{Introduction}

Morphometric studies have contributed greatly to our understanding of diabetic glomerular disease (1-4). Most of these studies have profiled structural changes that culminate in glomerular sclerosis in type I diabetes. Glomerular structure in type II diabetes has been studied less extensively, though this form of diabetes is the more common cause of renal failure. This is in part because patients of European ancestry usually develop type II diabetes late in life. Renal disease in these patients, who may also suffer from atherosclerotic vascular disease and hypertension, is not always attributable to diabetes $(5,6)$. In addition, the onset of type II diabetes is usually not well documented, and the dependence of renal injury on the duration of diabetes is therefore hard to assess.

Address correspondence to Timothy W. Meyer, Nephrology 111R, VA Palo Alto Healthcare System, 3801 Miranda Ave., Palo Alto, CA 94304. Phone: 415-852-3314; FAX: 415-852-3411.

Received for publication 11 April 1996 and accepted in revised form 19 November 1996.

The Journal of Clinical Investigation

Volume 99, Number 2, January 1997, 342-348
The current study sought to overcome these difficulties by examining glomerular structure in Pima Indians with type II diabetes. Pima Indians develop type II diabetes at an early age and are not afflicted by equally early development of hypertension, atherosclerosis, or nondiabetic renal disease $(7,8)$. In addition, the onset of diabetes and the magnitude of albuminuria in individual Pima subjects have been recorded in the course of ongoing epidemiologic studies. We were thus able to examine glomerular structure in Pima subjects in whom the duration of diabetes and the development of clinically apparent glomerular injury, reflected by albuminuria, were well documented. An initial aim was to determine if the sequence of glomerular structural changes in type II diabetes is similar to that observed in type I diabetes. A key structural finding in type I diabetes has been that progressive mesangial expansion contributes to loss of glomerular function $(3,4)$. The current study examined whether mesangial expansion follows the same pattern in type II diabetes. A second aim was to assess the potential contribution of visceral epithelial cell injury to the progression of diabetic glomerulopathy. The elaborately shaped visceral epithelial cell, or podocyte, appears to be incapable of replication in adult animals (9-11). Recent studies have further suggested that loss of podocytes imposes a burden on the remaining cells, which precipitates the development of glomerular sclerosis. These experimental findings prompted us to assess podocyte number in patients with diabetic glomerulopathy.

\section{Methods}

Renal biopsies were performed in 51 Pima Indians with type II diabetes. Subjects were recruited from among those participating in an ongoing study of renal function in type II diabetes $(12,13)$. For the purposes of the current study, subjects were grouped according to the duration of diabetes and the extent of glomerular injury as reflected by the urine albumin to creatinine $(\mathrm{A} / \mathrm{C})^{1}$ ratio. Because our purpose was to identify structural changes associated with the development of nephropathy, subjects with advanced renal insufficiency reflected by GFR values $<55 \mathrm{ml} / \mathrm{min}$ per $1.73 \mathrm{~m}^{2}$ were not enrolled. Renal function was assessed near the subjects' home in Arizona as previously described (12). A urine sample was collected for determination of the albumin to creatinine ratio and the GFR was measured as the urinary clearance of iothalamate during diuresis initiated by an oral water load. Biopsies were performed at the Stanford University Hospital within 6 mo of functional studies. When more than one functional study was performed within 6 mo of the biopsy, the results were averaged for correlation with the morphologic findings. Control tissue was obtained from eight living donor kidneys harvested at Stanford (14). The ethnic background of the control subjects was predomi-

1. Abbreviation used in this paper: $\mathrm{A} / \mathrm{C}$, albumin to creatinine. 
nantly Asian in three cases, European in three cases, and Hispanic in two cases.

Renal biopsy was performed under ultrasound guidance using a 14-gauge needle (Tru-Cut; Baxter Healthcare Corp., Deerfield, IL). Biopsy cores were embedded in Epon and sectioned serially at $2.5-\mu \mathrm{m}$ intervals. Every fourth section was mounted, four sections to a slide, and stained with toluidine blue so that the entire core could be viewed at $10-\mu \mathrm{m}$ intervals. An average of 92 sections were examined in each subject. Every other mounted section was photographed and a series of prints $(\sim 40 \times)$ were prepared for use as photographic maps of the serial sections from each core. Only glomeruli contained entirely within the core, and thus represented completely by serial profiles on the serial sections, were studied. An average of 20 (range, 5 to 49) of these complete glomeruli were then examined by light microscopy and categorized as sclerotic or "open." Glomeruli were classified as sclerotic if the midsection exhibited complete loss of capillary structure with solidification of the tuft. The prevalence of sclerotic glomeruli was multiplied by a factor that ranged from 0.79 to 0.86 in the different groups to correct for the tendency of sclerotic glomeruli, which are smaller than open glomeruli, to be included more often in biopsy cores. Mean values for the volumes of sclerotic and open glomeruli in each group were used to calculate the correction factor for that group based on the assumption that biopsy cores are cylindrical. The volumes of individual glomeruli in each subject were calculated from the areas of their midsections using the maximum planar area method of Lane et al. (15) and a computer-assisted morphometric unit (National Institutes of Health Image software). In a subset of patients, serial profiles of complete glomeruli were further examined to determine the prevalence of open glomeruli without proximal tubule connections ("atubular glomeruli") as described by Marcussen (16). The volume fraction of cortical interstitium was determined by point counting in five to seven sections spaced $80-\mu \mathrm{m}$ apart in each core (17). Diameters of proximal tubules were measured on tubule cross-sections whose centers fell within randomly chosen cortical fields $(\sim 20$ tubule cross-sections per subject). Measurements were made along the shortest axis through the center of the tubule section, and cell height was calculated as half the difference between the outer and luminal diameters.

Midsections of three open glomeruli from each biopsy were reembedded in Epon and thin-sectioned for examination by electron microscopy. Montage micrographs of whole profiles of each glomerulus $(\sim 1,600$ to $2,610 \times)$ were prepared for measurement of the surface densities of the peripheral capillary wall and epithelial-mesangial interface as described by Østerby and Gunderson (18). Glomerular basement membrane thickness was measured on five higher magnification electron micrographs $(\sim 9,400 \times)$ from each glomerulus using the orthogonal intercept method of Jensen et al. (19). Volume densities of glomerular components were determined by point counting on the montage micrographs (45 cases) or the higher power micrographs (14 cases). Absolute values for the volumes and areas of the components of glomerular structure were then calculated by multiplying the appropriate densities by values for the glomerular tuft volume. The average foot process width was determined on the higher power micrographs by dividing the number of slits overlying the peripheral capillary basement membrane by the length of the membrane in cross-section, and then multiplying by $\pi / 4$ to correct for variation in the angle of section relative to the long axis of the foot processes (20). The effects of altered foot process width and basement membrane thickness on capillary wall hydraulic permeability were estimated using the model of Drumond and Deen (21) and assuming that the resistance to filtration imposed by the endothelium is negligible.

The average number of podocytes per glomerulus was determined using the method of Weibel (22). The profile density $\left(N_{\mathrm{A}(\mathrm{Nepi})}\right)$ of the podocyte nuclei was first determined on the three montage glomerular profiles from each subject. The volume fraction $\left(V_{\mathrm{v}(\mathrm{Nepi})}\right)$ of podocyte nuclei within the glomerulus was next determined by dividing the total nuclear area by the glomerular profile area as determined by point counting. The number of podocytes per glomerulus was then obtained by multiplying glomerular volume by the numeric density $\left(N_{\mathrm{v}(\mathrm{EpiC})}\right)$ of the podocytes calculated as:

$N_{\mathrm{V}(\mathrm{EpiC})}=\frac{1}{\beta} \sqrt{\frac{\left(N_{\mathrm{A}(\mathrm{NEpi})}\right)^{3}}{V_{\mathrm{V}(\mathrm{NEpi})}}}$

where values for the shape constant $\beta$ are calculated based on the assumption that podocyte nuclei have a prolate spheroid shape $(9,22)$. Measurement of the axial ratios of 15 podocyte nuclear cross-sections in four cases from each group yielded values for $\beta$ of 1.45 in Pima Indians and 1.65 in living kidney donors used as controls. The average number of nonepithelial cells per glomerulus was determined using the same method with $\beta$ values of 1.50 and 1.56 obtained in Pima Indians and controls. The average volumes of individual podocytes and podocyte nuclei were estimated by dividing total glomerular podocyte volume and podocyte nuclear volume by the mean number of podocytes per glomerulus.

A one-factor ANOVA and Fisher's PLSD test for pairwise comparisons were used to compare findings in the study groups. Values for mesangial volume were logarithmically transformed for analysis. Results are given as the mean \pm SEM throughout. The study protocol was approved by the review boards of the participating institutions and by the Gila River Indian Community Tribal Council (Sacaton, AZ).

\section{Results}

Clinical data are summarized in Table I. Diabetic subjects were divided into four groups based on the urine $\mathrm{A} / \mathrm{C}$ ratio and the duration of diabetes. The first group had early diabetes defined by a disease duration of $\leq 6 \mathrm{yr}$ and an $\mathrm{A} / \mathrm{C}$ ratio of $\leq 30 \mathrm{mg} / \mathrm{g}$. The second group had long term normoalbuminuria defined by an $\mathrm{A} / \mathrm{C}$ ratio that remained $\leq 30 \mathrm{mg} / \mathrm{g}$ despite a disease duration $\geq 9 \mathrm{yr}$. This group was selected to have an average disease duration similar to that of the third group of subjects who had microalbuminuria defined by an A/C ratio of $30-300 \mathrm{mg} / \mathrm{g}$. The fourth group of subjects had clinical nephropathy defined by an $\mathrm{A} / \mathrm{C}$ ratio of $\geq 300 \mathrm{mg} / \mathrm{g}$. As expected, the duration of diabetes was greater in subjects with clinical nephropathy than in subjects with microalbuminuria. There were no significant differences in age, body weight, and $\mathrm{HbA}_{\mathrm{Ic}}$ among the diabetic groups. Blood pressure was slightly lower in subjects with long term normoalbuminuria than in those with early diabetes or clinical nephropathy. A tendency toward elevation in blood pressure with the development of clinical nephropathy may have been obscured by the use of antihypertensive medications. 4 of the 12 subjects with nephropathy were using antihypertensive agents as compared with only 2 of 17 subjects with microalbuminuria and no subjects in the other groups. 8 of 12 subjects with clinical nephropathy, however, remained normotensive with mean blood pressure values averaging $99 \pm 4 \mathrm{mmHg}$. Values for GFR in subjects with early diabetes, long term normoalbuminuria, and microalbuminuria were elevated in comparison with values obtained using the same technique in normal subjects from our local area (23). A significant reduction in GFR was associated with the onset of clinical nephropathy, but GFR values in subjects with nephropathy remained within the range observed in normal controls. Functional evaluation of kidney donors who provided control tissue for the current study was limited to the finding of normal values for serum creatinine and 24 -h urine protein excretion $(<150 \mathrm{mg} / \mathrm{d})$.

Features of kidney structure are summarized in Table II. 


\begin{tabular}{|c|c|c|c|c|c|}
\hline & Early diabetes & Long term normoalbuminuria & Microalbuminuria & Clinical nephropathy & Control \\
\hline $\mathrm{A} / \mathrm{C}(\mathrm{mg} / \mathrm{g})$ & $11 \pm 3$ & $14 \pm 2$ & $81 \pm 9$ & $2778 \pm 770$ & - \\
\hline $\operatorname{Sex}(m / f)$ & $6 / 4$ & $6 / 6$ & $6 / 11$ & $7 / 5$ & $3 / 5$ \\
\hline Age (yr) & $40 \pm 4$ & $43 \pm 3$ & $41 \pm 3$ & $47 \pm 3^{*}$ & $34 \pm 3$ \\
\hline Duration of diabetes (yr) & $3 \pm 1$ & $14 \pm 1$ & $13 \pm 1$ & $19 \pm 2$ & - \\
\hline Weight $(\mathrm{kg})$ & $90 \pm 4 *$ & $80 \pm 4$ & $89 \pm 4 *$ & $92 \pm 5^{*}$ & $69 \pm 5$ \\
\hline $\mathrm{HbA}_{\mathrm{Ic}}(\%)$ & $9 \pm 1$ & $11 \pm 1$ & $10 \pm 1$ & $10 \pm 1$ & - \\
\hline Mean arterial pressure $(\mathrm{mmHg})$ & $95 \pm 2$ & $86 \pm 2^{\ddagger}$ & $92 \pm 2$ & $97 \pm 3^{\S}$ & $92 \pm 2$ \\
\hline Creatinine $(\mathrm{mg} / \mathrm{dl})$ & $0.67 \pm 0.03^{*}$ & $0.64 \pm 0.02 *$ & $0.62 \pm 0.04 *$ & $0.92 \pm 0.08^{\ddagger \S} \|$ & $0.89 \pm 0.10$ \\
\hline $\operatorname{GFR}\left(\mathrm{ml} / \mathrm{min}\right.$ per $\left.1.73 \mathrm{~m}^{2}\right)$ & $143 \pm 15$ & $143 \pm 8$ & $156 \pm 10$ & $103 \pm 12^{\ddagger \S} \|$ & - \\
\hline
\end{tabular}

${ }^{*} P<0.05$ Pima groups vs. controls, ${ }^{\ddagger} P<0.05$ vs. early diabetes, ${ }^{\S} P<0.05$ vs. long term normoalbuminuria, and $\| P<0.05$ vs. microalbuminuria.

The development of clinical nephropathy was associated with a marked increase in the prevalence of global sclerosis. The increase in global sclerosis was associated with typical changes in open glomeruli, including prominent mesangial expansion and deposition of hyaline material that accumulated in large nodules in some glomeruli. Collapse of capillary lumina and focal adhesions of the tuft to Bowman's capsule were observed along with mesangial expansion and hyaline deposition in some glomerular profiles. Increased global sclerosis in subjects with clinical nephropathy was also accompanied by an increase in the volume fraction of the cortical interstitium ( $\mathrm{Vv}$ interstitium) to $28 \pm 2 \%$ as compared with $22 \pm 2 \%$ in subjects with early diabetes. Examination of serial sections in six patients with clinical nephropathy revealed that this moderate increase in interstitial volume fraction was not associated with the appearance of atubular glomeruli.

The appearance of glomerular injury in Pima Indians with type II diabetes was preceded by an increase in glomerular volume. Glomeruli were more than twice as large in Pima Indians with early diabetes as in living kidney donors from the Stanford area. Glomeruli were even larger in subjects with long term diabetes, but there was no difference between subjects with microalbuminuria as compared with subjects with diabetes of equal duration and normoalbuminuria. Of note, increases in glomerular volume observed in diabetic Pima Indians were not associated with parallel increases in proximal tubule diameter or cell height. The development of clinical nephropathy was associated with a further increase in glomerular volume to a value $>50 \%$ greater than that seen in early diabetes. Measurements of glomerular ultrastructure showed that this increase in total glomerular volume was largely attributable to an increase in mesangial volume, as illustrated in Fig. 1. The volume of the mesangium expressed as a percentage of the total glomerular volume ( Vv mesangium) was increased in subjects with clinical nephropathy in comparison with all other groups, and exceeded by twofold that in subjects with early diabetes. Because glomerular volume increased along with mesangial volume, however, this degree of mesangial expansion did not reduce the peripheral capillary surface area $\left(\mathrm{S}_{\mathrm{PCW}}\right)$ below the level seen in early diabetes. Maintenance of a stable peripheral capillary surface area during the development of clinical nephropathy was associated with maintenance of near constant values for the average volume per glomerulus of capillary lumina, epithelial cells, and endothelial cells (values not shown). Though it was not attended by a reduction in peripheral capillary surface area, the development of clinical nephropathy was associated with changes in the structure of the capillary wall. Subjects with clinical nephropathy exhibited widening of epithelial cell foot processes and thickening of the glomerular basement membrane. Application of the theoretical model of Drumond and Deen (21) suggested that these changes were of sufficient magnitude to reduce capillary wall hydraulic permeability. The model yielded a hydraulic permeability value of $2.4 \pm 0.2 \times 10^{-9} \mathrm{~m} / \mathrm{s}$ per $\mathrm{Pa}$ in subjects with clin-

Table II. Summary of Morphometric Studies

\begin{tabular}{|c|c|c|c|c|c|}
\hline & Early diabetes & Long term normoalbuminuria & Microalbuminuria & Clinical nephropathy & Control \\
\hline Global sclerosis (\%) & $3 \pm 1$ & $5 \pm 3$ & $5 \pm 1$ & $19 \pm 4 * \notin \S \|$ & $6 \pm 3$ \\
\hline Vv interstitium (\%) & $22 \pm 3$ & $23 \pm 2 *$ & $25 \pm 1 *$ & $28 \pm 2 * \ddagger$ & $17 \pm 1$ \\
\hline Glomerular volume $\left(\times 10^{6} \mu \mathrm{m}^{3}\right)$ & $5.4 \pm 0.3^{*}$ & $6.9 \pm 0.5^{* \ddagger}$ & $6.9 \pm 0.5^{* \dot{亠}}$ & $8.5 \pm 0.5 * \div \S \|$ & $2.6 \pm 0.3$ \\
\hline Proximal tubule diameter $(\mu \mathrm{m})$ & $46 \pm 2$ & $47 \pm 2$ & $47 \pm 2$ & $47 \pm 2$ & $46 \pm 2$ \\
\hline Proximal tubule cell height $(\mu \mathrm{m})$ & $12 \pm 1$ & $12 \pm 1$ & $11 \pm 1$ & $12 \pm 1$ & $12 \pm 1$ \\
\hline Vv mesangium (\%) & $21 \pm 2$ & $25 \pm 2 *$ & $28 \pm 2 * \ddagger$ & $42 \pm 5^{*} \notin \S \|$ & $17 \pm 1$ \\
\hline $\mathrm{S}_{\mathrm{PCW}}\left(10^{3} \mu \mathrm{m}^{2}\right)$ & $535 \pm 46^{*}$ & $646 \pm 56^{*}$ & $596 \pm 55^{*}$ & $511 \pm 64 *$ & $312 \pm 41$ \\
\hline GBM thickness (nm) & $427 \pm 37$ & $500 \pm 30 *$ & $504 \pm 19 *$ & $606 \pm 44 * \ddagger 8 \|$ & $329 \pm 19$ \\
\hline Foot process width $(\mathrm{nm})$ & $650 \pm 23$ & $696 \pm 25$ & $645 \pm 31$ & $835 \pm 51^{\ddagger \S \|}$ & $737 \pm 40$ \\
\hline
\end{tabular}

${ }^{*} P<0.05$ Pima groups vs. controls, ${ }^{\ddagger} P<0.05$ vs. early diabetes, ${ }^{\S} P<0.05$ vs. long term normoalbuminuria, and $\| P<0.05$ vs. microalbuminuria. Abbreviations: Vv interstitium, volume fraction of the cortical interstitium; Vv mesangium, mesangial volume fraction; S $\mathrm{PCW}$, peripheral capillary surface area; and GBM thickness, glomerular basement membrane thickness. 


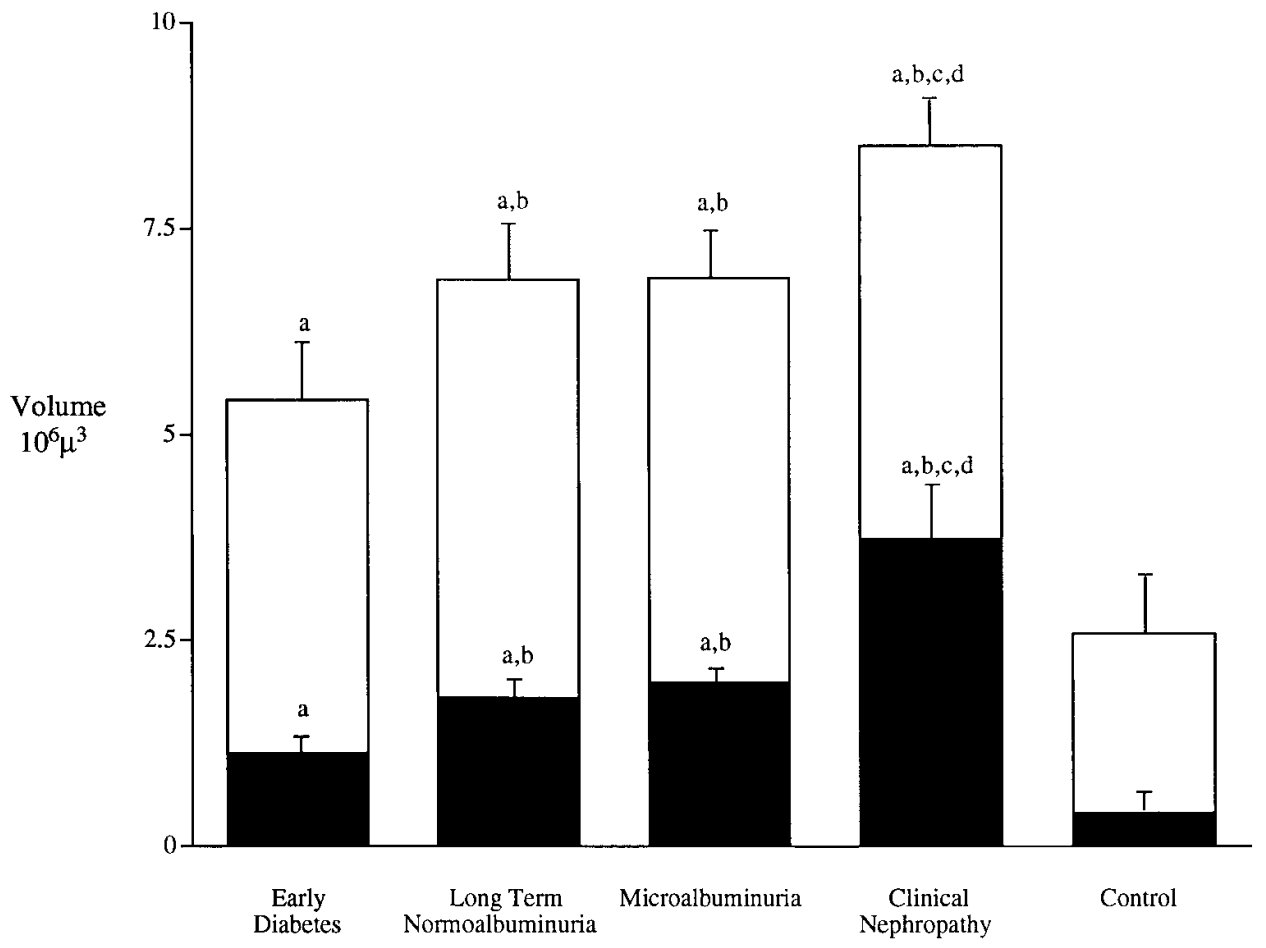

Figure 1. Total glomerular volume (whole bar) and mesangial volume (filled portion) in the five study groups. a, $P<0.05$, Pima groups vs. controls; $\mathrm{b}, P<0.05$ vs. early diabetes; c, $P<0.05$ vs. long term normoalbuminuria; $\mathrm{d}, P<0.05$ vs. microalbuminuria. ical nephropathy. This value was significantly reduced as compared with the values of $3.1 \pm 0.1,2.8 \pm 0.1,2.9 \pm 0.1$, and $3.0 \pm 0.1 \times 10^{-9} \mathrm{~m} / \mathrm{s}$ per Pa calculated for Pima subjects with early diabetes, long term normoalbuminuria, microalbuminuria, and controls subjects, respectively.

Subjects with microalbuminuria did not exhibit the marked changes in glomerular ultrastructure observed in subjects with clinical nephropathy. Foot process width was not different in subjects with microalbuminuria than in subjects with early diabetes or subjects with long term diabetes who remained normoalbuminuric. Values for mesangial volume fraction and glomerular basement membrane thickness were higher in subjects with microalbuminuria than in subjects with early diabetes, but only the difference in mesangial volume fraction reached statistical significance. Moreover, values for these structural parameters were nearly identical in subjects with microalbuminuria and in subjects who had diabetes for a similar period of time but remained normoalbuminuric. Subjects with microalbuminuria remained structurally indistinguishable from those with long term diabetes and normoalbuminuria if the defini- tion of microalbuminuria were changed to include subjects with $\mathrm{A} / \mathrm{C}$ ratios $>60 \mathrm{mg} / \mathrm{g}(n=11)$, instead of subjects with $\mathrm{A} / \mathrm{C}$ ratios greater than $30 \mathrm{mg} / \mathrm{g}(n=17)$.

Measurements of glomerular cell number are summarized in Table III. A marked reduction in the number of podocytes per glomerular tuft was associated with mesangial expansion and altered capillary wall structure in subjects with clinical nephropathy. Because glomerular volume increased with the development of nephropathy, the reduction in podocyte number was accompanied by an even more marked reduction in podocyte density expressed as the number of podocytes per unit tuft volume. Calculation of the average surface area covered by individual podocytes confirmed that reduction in podocyte number was not due simply to obliteration of normal tuft structure by the expanding mesangium. This calculation showed that loss of podocytes in patients with clinical nephropathy was associated with an increase in the surface area covered by each remaining cell. Loss of podocytes was also accompanied by increases in the volumes of remaining podocytes and their nuclei. Values for the number of nonepi-

Table III. Quantitation of Epithelial and Nonepithelial Cells in the Glomerular Tuft

\begin{tabular}{|c|c|c|c|c|c|}
\hline & Early diabetes & Long term normoalbuminuria & Microalbuminuria & Clinical nephropathy & Control \\
\hline Epithelial cell number & $476 \pm 32$ & $512 \pm 41$ & $464 \pm 34 *$ & $351 \pm 21 * \ddagger \S \|$ & $575 \pm 45$ \\
\hline Epithelial cell density (No. $/ 10^{6} \mu \mathrm{m}^{3}$ ) & $89 \pm 6^{*}$ & $75 \pm 3 *$ & $70 \pm 5^{*}$ & $43 \pm 3 * \ddagger \S \|$ & $235 \pm 25$ \\
\hline Area per epithelial cell $\left(10^{3} \mu \mathrm{m}^{2}\right)$ & $1.8 \pm 0.1 *$ & $1.9 \pm 0.1 *$ & $2.0 \pm 0.1 *$ & $2.8 \pm 0.3 * \div \S \|$ & $0.9 \pm 0.1$ \\
\hline Epithelial cell volume $\left(10^{3} \mu \mathrm{m}^{3}\right)$ & $3.1 \pm 0.3^{*}$ & $2.9 \pm 0.2 *$ & $3.1 \pm 0.2 *$ & $4.1 \pm 0.3 * \frac{i+}{\S \|}$ & $1.35 \pm 0.14$ \\
\hline Epithelial cell nuclear volume $\left(\mu \mathrm{m}^{3}\right)$ & $249 \pm 13^{*}$ & $228 \pm 9 *$ & $230 \pm 10 *$ & $281 \pm 17 * 8 \|$ & $158 \pm 10$ \\
\hline Nonepithelial cell number & $2294 \pm 204 *$ & $2967 \pm 256^{*}$ & $3153 \pm 288^{* *}$ & $4153 \pm 266 * \$ \$ \|$ & $1118 \pm 122$ \\
\hline Nonepithelial cell density (No. $/ 10^{6} \mu \mathrm{m}^{3}$ ) & $426 \pm 34$ & $427 \pm 19$ & $454 \pm 24$ & $491 \pm 21$ & $436 \pm 32$ \\
\hline
\end{tabular}

${ }^{*} P<0.05$ Pima groups vs. controls, ${ }^{\ddagger} P<0.05$ vs. early diabetes, ${ }^{\S} P<0.05$ vs. long term normoalbuminuria, and $\| P<0.05$ vs. microalbuminuria. Abbreviations: Area per epithelial cell, average peripheral capillary and mesangial-epithelial surface covered by each epithelial cell. 
thelial cells exhibited a markedly different pattern. The number of nonepithelial cells increased in proportion with glomerular growth in subjects with long term diabetes with normoalbuminuria and microalbuminuria. A further increase in nonepithelial cell number accompanied enlargement of nonsclerosed glomeruli in patients with clinical nephropathy, so that the density of nonepithelial cells remained the same in each group.

Measurements of cell number also revealed a prominent difference between diabetic Pima Indians and Stanford area controls. Despite having much larger glomerular volumes and surface areas, the Pima Indians had no more podocytes per glomerulus. Podocyte density was thus decreased and the surface covered by individual podocytes was increased in the Pima groups as compared with the control group. The increase in surface area covered was accompanied by increases in podocyte cell volume and nuclear volume. The difference in nuclear structure was readily apparent on inspection of low power electron micrographs. Podocyte nuclei in Pima subjects were not only larger, but exhibited more diffuse chromatin and prominent nucleoli, than podocyte nuclei in Stanford area controls. Nonepithelial cells again presented a different pattern. Large glomerular size in the diabetic Pima Indians was accompanied by an increase in the number of nonepithelial cells so that cell number per unit volume was the same as in the controls.

\section{Discussion}

Proteinuria in European subjects with type II diabetes is associated with a variety of glomerular lesions, some of which are not attributable to diabetes $(5,6)$. In the current study, albuminuria in Pima Indians with type II diabetes was found to be associated exclusively with structural changes similar to those described in type I diabetes $(3,4)$. Presumably, glomerular injury is more homogenous in Pima Indians than in European subjects with type II diabetes because the Pima Indians develop type II diabetes earlier in life and usually do not become hypertensive or suffer atherosclerotic vascular disease before diabetic nephropathy is established (8).

Pima Indians with early diabetes had high values for GFR and exhibited marked glomerular enlargement in comparison with local controls. Glomerular capillary surface area was increased along with glomerular volume while the structure of the capillary wall, as assessed by determination of basement membrane thickness and foot process width, remained normal. These findings are consistent with the suggestion first made by Kroustrup et al. (1) and Østerby et al. (24) that an enlarged capillary surface area contributes to elevation of the GFR early in the course of diabetes. It should be noted that micropuncture studies have suggested that glomerular hyperfiltration in diabetic rats is associated with an increase in glomerular pressure, while the glomerular ultrafiltration coefficient remains normal despite an increase in glomerular volume (25, 26). To date, no structural rearrangement has been identified that can resolve the apparent paradox of a constant ultrafiltration coefficient in the face of an increased capillary surface area.

Pima Indians with diabetes of longer duration but without clinical nephropathy exhibited a further increase in glomerular volume. A disproportionate share of the overall increase in glomerular volume was attributable to enlargement of the me- sangium. Capillary surface area, however, remained high and glomerular basement membrane thickness and epithelial cell foot process width were not significantly increased. Preservation of capillary surface area and near normal capillary wall structure were associated with stability of the GFR. In the current study, microalbuminuria could not be distinguished from normoalbuminuria on structural grounds. Epidemiologic studies have shown that microalbuminuria predicts the development of clinical nephropathy in Pima Indians as well as in European subjects with types I and II diabetes $(27,28)$. These observations have prompted investigation of whether microalbuminuria reveals the presence of glomerular structural injury. Studies in type I diabetes have suggested that microalbuminuria is associated with early mesangial expansion and glomerular basement membrane thickening (29-32). The extent of these changes has been modest, however, and subjects with microalbuminuria have in most cases not been compared with subjects remaining normoalbuminuric after diabetes of equal duration. In the current study, mesangial volume fraction and basement membrane thickness were slightly higher in subjects with microalbuminuria than in subjects with early diabetes, but almost identical in subjects with microalbuminuria and subjects who had diabetes for a similar period but remained normoalbuminuric. It is possible that a study including larger numbers of subjects and comparing fewer groups would have detected significant structural differences between microalbuminuria and long term diabetes with normoalbuminuria. In addition, subtle changes in capillary structure that are not detected by current morphometric techniques may account for the appearance of microalbuminuria in subjects in whom mesangial volume fraction and basement membrane thickness remain the same as in subjects with normoalbuminuria (32).

In contrast with microalbuminuria, clinical nephropathy was associated with extensive structural changes. The most prominent of these was global sclerosis. Nephron loss due to glomerular sclerosis was associated with an increase in the interstitial volume fraction. The extent of interstitial expansion was modest however, and atubular glomeruli were not observed. These findings suggest that glomerular injury is largely responsible for the reduction in GFR that accompanies the development of clinical nephropathy.

Glomerular sclerosis in subjects with clinical nephropathy was associated with an increase in the volume of remaining open glomeruli, as has been observed in type I diabetes (24). This increase in glomerular volume may have been caused both by mesangial expansion, which by itself would be expected to reduce capillary surface area, and by compensatory hypertrophy, which would tend to increase the capillary surface in remnant glomeruli. The current results are consistent with the suggestion that the combined effect of these processes is to maintain stable capillary surface area while glomeruli enlarge during the initial phase of clinical nephropathy (33). While capillary surface area per glomerulus remained stable in subjects with clinical nephropathy, the structure of the capillary wall was markedly altered. In particular, the glomerular basement membrane was thickened and the foot processes of the overlying podocytes were broadened. Theoretical modeling suggests that the effect of both these changes, which have previously been described in type I diabetes, is to reduce glomerular ultrafiltration capacity per unit area of capillary surface (21). In the current study, the reduction in GFR associated with the development of clinical nephropathy could thus be at- 
tributed to a reduction in total capillary surface area caused by glomerular sclerosis together with changes in the capillary wall structure of remaining open glomeruli.

The association of modest structural changes with microalbuminuria and marked changes with clinical nephropathy implies that the progression of diabetic glomerular injury is not linear with time. As summarized in Fig. 1 and Table II, subjects with microalbuminuria exhibited increases in mesangial volume and basement membrane thickness of $\sim 75$ and $15 \%$ at an average duration of diabetes of $13 \mathrm{yr}$. Subjects with clinical nephropathy exhibited increases in these parameters of $\sim 225$ and $40 \%$ at an average duration of diabetes that was only 6 yr longer. The design of the current study was crosssectional, but longitudinal studies have shown that this interval is typical of the time required for progression from microalbuminuria to macroalbuminuria in the Pima population (28). It thus appears that mesangial expansion and basement membrane thickening proceed slowly through the phase of microalbuminuria, and then accelerate in conjunction with the appearance of foot process widening and glomerular sclerosis as clinical nephropathy develops. Results of the current study suggest that this late acceleration of glomerular injury could be precipitated by loss of visceral epithelial cells. In the normal glomerulus, the visceral epithelial cell, or podocyte, is responsible for production of the outer portion of the basement membrane and for maintenance of the filtration slit structure that prevents protein escape from the glomerular capillaries $(34,35)$. These crucial functions are carried out by a complex array of interdigitating foot processes that are distant from the cell body and protein synthetic apparatus. Studies in rats with reduced nephron number have shown that the elaborately shaped podocyte is incapable of replication $(9,10)$. These studies have shown further that podocytes suffer progressive injury when obliged to cover an increased surface area. Podocyte injury in this setting is characterized initially by proteinuria and widening of foot processes and culminates in glomerular sclerosis. In the current study, development of clinical nephropathy was associated with a reduction in the number of podocytes per glomerulus, while the total surface area covered by the podocytes did not change. Remaining podocytes were obliged to grow and to extend their foot processes to maintain the area covered. The experimental studies cited above suggest that these podocyte structural changes could precipitate development of glomerular sclerosis in diabetic patients with clinical nephropathy.

A number of mechanisms could account for podocyte loss in diabetes. First, it has been suggested that sustained mechanical stress associated with glomerular hypertrophy and hypertension causes podocyte injury (11). Second, it has been noted that the podocyte resembles the peripheral neuron in its inability to replicate and its obligation to supply elongated processes from a central cell body (34). The mechanisms responsible for podocyte injury in diabetic glomerulopathy could thus be related to those responsible for diabetic neuropathy. Finally, mesangial expansion beyond some critical point can presumably cause closure of capillary loops and obliteration of podocytes. At present, these potential mechanisms of injury cannot be distinguished by morphologic techniques and so remain hypothetical. Nevertheless, the current data suggest that loss of podocytes is associated with acceleration of glomerular structural injury in patients advancing from microalbuminuria to clinical nephropathy.
An interesting finding of the current study was that Pima Indians with early diabetes had larger glomeruli but no more podocytes per glomerulus than nondiabetic subjects from our local area. Pima Indians without diabetes were not subjected to biopsy, so the data do not show whether these differences are due to diabetes or to ethnic differences between the Pima Indians and local controls. Previous studies suggest, however, that a considerable portion of the difference is ethnic. Autopsy studies have shown that nondiabetic Pima Indians have larger glomeruli than subjects of European ancestry (36). Moreover, glomerular volume in Pima Indians was found to be greater than would be predicted using the relation of glomerular size to body surface area derived in European subjects (37). Glomerular ultrastructure cannot be assessed in the autopsy material, so podocyte number in nondiabetic Pima Indians has not been determined. It seems unlikely, however, that podocyte number in Pima Indians falls when diabetes develops and then remains stable over the first decade of the disease, only to decline again as clinical nephropathy develops. Rather, low podocyte number per unit glomerular volume and capillary surface area would appear to be a characteristic of the Pima population. It is tempting to speculate that this characteristic contributes to the unusually high incidence of clinical nephropathy in Pima Indians with diabetes (38).

In summary, findings of the current study are consistent with the conclusion that the same pathologic processes cause glomerular injury in types I and II diabetes $(39,40)$. Structural changes in subjects with microalbuminuria are modest, however, and do not differentiate these subjects from those who remain normoalbuminuric after diabetes of equal duration. Structural changes in subjects with clinical nephropathy are more pronounced. The reduction in GFR that accompanies the development of clinical nephropathy can be accounted for by loss of glomeruli due to sclerosis and changes in the capillary wall structure of remaining open glomeruli. Subjects with clinical nephropathy also exhibit a reduction in the number of podocytes per glomerulus and an increase in the surface area that must be covered by remaining podocytes. These findings suggest that podocyte loss contributes to the progression of diabetic nephropathy.

\section{Acknowledgments}

The authors are indebted to Lois Jones for arranging the biopsy studies, to Helen Kwan for preparation of tissue sections, and to Jon Kosek for diagnostic review of biopsies.

This study was supported by the National Institutes of Health (NIH) (DK-43597 and DK-72291), the Juvenile Diabetes Foundation, the Baxter Extramural Grant Program, and the Research Service of the Veterans Administration. M.E. Pagtalunan was supported by an NIH training grant (DK-07357).

\section{References}

1. Kroustrup, J.P., H.J.G. Gundersen, and R. Østerby. 1977. Glomerular size and structure in diabetes mellitus. Diabetologia. 13:207-210.

2. Mauer, S.M., M.W. Steffes, E.L. Ellis, D.E.R. Sutherland, D.M. Brown, and F.C. Goetz. 1984. Structural-functional relationships in diabetic nephropathy. J. Clin. Invest. 74:1143-1155.

3. Østerby, R., H.-H. Parving, E. Hommel, H.E. Jørgensen, and H. Løkkegaard. 1990. Glomerular structure and function in diabetic nephropathy. Early to advanced stages. Diabetes. 39:1057-1063.

4. Mauer, S.M. 1994. Structural-functional correlations of diabetic nephropathy. Kidney Int. 45:612-622.

5. Parving, H.-H., M.-A. Gall, P. Skøtt, H.E. Jørgensen, H. Løkkegaard, F. 
Jørgensen, B. Nielsen, and S. Larsen. 1992. Prevalence and causes of albuminuria in non-insulin-dependent diabetic patients. Kidney Int. 41:758-762.

6. Gambara, V., G. Mecca, G. Remuzzi, and T. Bertani. 1993. Heterogeneous nature of renal lesions in type II diabetes. J. Am. Soc. Nephrol. 3:14581466.

7. Knowler, W.C., D.J. Pettitt, M.F. Saad, and P.H. Bennett. 1990. Diabetes mellitus in the Pima Indians: Incidence, risk factors, and pathogenesis. Diabetes Metab. Rev. 6:1-27.

8. Nelson, R.G., W.C. Knowler, D.R. McCance, M.L. Sievers, D.J. Pettitt, M.A. Charles, R.L. Hanson, Q.Z. Liu, and P.H. Bennett. 1993. Determinants of end-stage renal disease in Pima Indians with type 2 (non-insulin-dependent) diabetes mellitus and proteinuria. Diabetologia. 36:1087-1093.

9. Fries, J.W.U., D.J. Sandstrom, T.W. Meyer, and H.G. Rennke. 1989. Glomerular hypertrophy and epithelial cell injury modulate progressive glomerulosclerosis in the rat. Lab. Invest. 60:205-218.

10. Nagata, M., and W. Kriz. 1992. Glomerular damage after uninephrectomy in young rats. II. Mechanical stress on podocytes as a pathway to sclerosis. Kidney Int. 42:148-160.

11. Kretzler, M., I. Koeppen-Hagemann, and W. Kriz. 1994. Podocyte damage is a critical step in the development of glomerulosclerosis in the uninephrectomised-desoxycorticosterone hypertensive rat. Virchows Arch. 425:181-193.

12. Myers, B.D., R.G. Nelson, G.W. Williams, P.H. Bennett, S.A. Hardy, R.L. Berg, N. Loon, W.C. Knowler, and W.E. Mitch. 1991. Glomerular function in Pima Indians with non-insulin-dependent diabetes mellitus of recent onset. J. Clin. Invest. 88:524-530.

13. Myers, B.D., R.G. Nelson, M. Tan, G.J. Beck, P.H. Bennett, W.C. Knowler, K. Blouch, and W.E. Mitch. 1995. Progression of overt nephropathy in non-insulin-dependent diabetes. Kidney Int. 47:1781-1789.

14. Pagtalunan, M.E., R. Oberbauer, M. Haas, M. Barlan, G. Mayer, J.L. Olson, and T.W. Meyer. 1996. Atubular glomeruli in patients with chronic allograft rejection. Transplantation (Baltimore). 61:1166-1171.

15. Lane, P.H., M.W. Steffes, and S.M. Mauer. 1992. Estimation of glomerular volume. A comparison of four methods. Kidney Int. 41:1085-1089.

16. Marcussen, N. 1992. Biology of disease. Atubular glomeruli and the structural basis for chronic renal failure. Lab. Invest. 66:265-284.

17. Lafayette, R.A., G. Mayer, and T.W. Meyer. 1993. The effects of blood pressure reduction on cyclosporine nephrotoxicity in the rat. J. Am. Soc. Nephrol. 3:1892-1899.

18. Østerby, R., and H.J.G. Gundersen. 1980. Fast accumulation of basement membrane material and the rate of morphologic changes in acute experimental diabetic glomerular hypertrophy. Diabetologia. 18:493-500.

19. Jensen, E.B., H.J.G. Gundersen, and R. Østerby. 1979. Determination of membrane thickness distribution from orthogonal intercepts. J. Microsc. (Oxf.) 115:19-33.

20. Pagtalunan, M.E., R. Rasch, H.G. Rennke, and T.W. Meyer. 1995. Morphometric analysis of effects of angiotensin II on glomerular structure in rats. Am. J. Physiol. 268:F82-F88.

21. Drumond, M.C., and W.M. Deen. 1994. Structural determinants of glomerular hydraulic permeability. Am. J Physiol. 266:F1-F12.

22. Weibel, E.R. 1979. Stereological Methods. Practical Methods for Biological Morphometry. Vol. 1. Academic Press, Inc., London. 40-116.

23. Ting, R.H., B. Krystal, and B.D. Myers. 1994. The biophysical basis of hypofiltration in nephrotic humans with membranous nephropathy. Kidney Int. 45:390-397.

24. Østerby, R., H.-H. Parving, G. Nyberg, E. Hommel, H.E. Jørgensen, H. Løkkegaard, and C. Svalander. 1988. A strong correlation between glomerular filtration rate and filtration surface in diabetic nephropathy. Diabetologia. 31 $265-270$.

25. Hostetter, T.H., J.L. Troy, and B.M. Brenner. 1981. Glomerular hemodynamics in experimental diabetes mellitus. Kidney Int. 19:410-415.

26. Østerby, R., and H.J.G. Gunderson. 1980. Fast accumulation of basement membrane material and the rate of morphological changes in acute experimental diabetic glomerular hypertrophy. Diabetologia. 18:493-500.

27. Mogensen, C.E. 1990. Prediction of clinical diabetic nephropathy in DDM patients. Diabetes. 39:761-767.

28. Nelson, R.G., W.C. Knowler, D.J. Pettitt, M.F. Saad, M.A. Charles, and P.H. Bennett. 1991. Assessment of risk of overt nephropathy in diabetic patients from albumin excretion in untimed urine specimens. Arch. Intern. Med. 151:1761-1765.

29. Chavers, B.M., R.W. Bilous, E.N. Ellis, M.W. Steffes, and S.M. Mauer. 1989. Glomerular lesions and urinary albumin excretion in type I diabetes without overt proteinuria. N. Engl. J. Med. 320:966-970.

30. Walker, J.D., C.F. Close, S.L. Jones, M. Rafftery, H. Keen, G. Viberti, and R. Østerby. 1992. Glomerular structure in type-1 diabetic patients with normo-and microalbuminuria. Kidney Int. 41:741-748.

31. Banstad, H.-J., R. Østerby, K. Dahl-Jorgensen, K.J. Berg, A. Hartmann, G. Nyberg, S. Frahm Bjorn, and K.F. Hannsen. 1993. Early glomerulopathy is present in young, type 1 diabetic patients with microalbuminuria. Diabetologia. 36:523-529.

32. Østerby, R. 1995. Microalbuminuria in diabetes mellitus-is there a structural basis? Nephrol. Dial. Transplant. 10:12-14.

33. Austen, S.M., J.S. Lieberman, L.D. Newton, M. Mejia, W.A. Peters, and B.D. Myers. 1993. Slope of serial glomerular filtration rate and the progression of diabetic glomerular disease. J. Am. Soc. Nephrol. 3:1358-1370.

34. Rennke, H.G. 1994. How does glomerular epithelial cell injury contribute to progressive glomerular damage? Kidney Int. 45:S58-S63.

35. Kerjaschki, D. 1994. Dysfunctions of cell biological mechanisms of visceral epithelial cells (podocytes) in glomerular diseases. Kidney Int. 45:300-313.

36. Schmidt, K., C. Pesce, Q. Liu, R.G. Nelson, P.H. Bennett, H. Karnitschnig, L.J. Striker, and G.E. Striker. 1992. Large glomerular size in Pima Indians: lack of change with diabetic nephropathy. J. Am. Soc. Nephrol. 3:229-235.

37. Nyengaard, J.R., and T.F. Bendtsen. 1992. Glomerular number and size in relation to age, kidney weight, and body surface in normal man. Anat. Rec. 232:194-201.

38. Kunzelman, C.L., W.C. Knowler, D.F. Pettitt, and P.H. Bennett. 1989 Incidence of proteinuria in type 2 diabetes mellitus in the Pima Indians. Kidney Int. 35:681-687.

39. Hayashi, H., R. Karasawa, H. Inn, T. Saitou, M. Ueno, S. Nishi, Y. Suzuki, S. Ogino, Y. Maruyama, Y. Kouda, et al., 1992. An electron microscopic study of glomeruli in Japanese patients with non-insulin dependent diabetes mellitus. Kidney Int. 41:749-757.

40. Østerby, R., M.-A. Gall, A. Schmitz, F.S. Nielsen, G. Nyberg, and H.-H. Parving. 1993. Glomerular structure and function in proteinuric type 2 (noninsulin-dependent) diabetic patients. Diabetologia. 36:1064-1070. 